\title{
Cerebral Microdialysis: Going Deep into Brain Biochemistry for a Better Understanding of Pathomechanisms of Acute Brain Injury
}

\author{
Deepak Kumar Gupta ${ }^{1}$ Anna Teresa Mazzeo ${ }^{2}$ \\ ${ }^{1}$ Department of Neurosurgery, All India Institute of Medical \\ Sciences, New Delhi, India \\ 2 Department of Surgical Sciences, University of Torino, Torino, Italy \\ Indian J Neurosurg 2017;6:86-94.
}

\begin{abstract}
Address for correspondence Deepak Kumar Gupta, MD, Department of Neurosurgery, All India Institute of Medical Sciences, New Delhi 110029, India (e-mail: drdeepakgupta@gmail.com).
\end{abstract}

\begin{abstract}
Patients with traumatic brain injury (TBI) have variable outcomes subject to intracranial hypertension, ischemia, hypotension, and various biochemical alterations. Multimodality neurointensive monitoring is standard of care for decision making in all severe TBI cases at present in most neurotrauma units. Neuromonitoring tools, such as cerebral microdialysis,

Keywords

- cerebral microdialysis

- severe traumatic brain injury

- decompressive craniectomy

- ischemia

- mitochondrial dysfunction may contribute to understanding the pathomechanisms of acute brain injury, provide an early warning of secondary cerebral deterioration (therapeutic window of opportunity), and help in selecting the patients most suitable to benefit from this very invasive surgical rescue therapy. Cerebral microdialysis can be used as a measure of the effect of decompressive craniectomy on neurochemistry derangement and brain energy crisis jeopardizing the injured brain. Present review discusses the cerebral microdialysis, its usefulness, and role in prognostication of severe TBIs, especially in patients postdecompressive craniectomy. The authors also discuss the current consensus guidelines on usage of cerebral microdialysis in TBI patients.
\end{abstract}

\section{Introduction}

Patients with traumatic brain injury (TBI) have variable outcomes subject to intracranial hypertension, ischemia, hypotension, and various biochemical alterations. Intracranial pressure (ICP) monitoring is indicated in all severe head injury cases with detectable lesions by computed tomography (CT) scan of the head as it has been shown that patients with ICP $>20 \mathrm{~mm} \mathrm{Hg}$ have poor outcome if not subjected to medical/surgical management for intractable intracranial hypertension. Despite adequate decompression for raised ICP, a subset of patients remain who continue to deteriorate and have poor neurologic outcome at long-term follow-up. This is being attributed to ongoing biochemical changes in brain tissue (secondary insult/ongoing insults) even after decompressive craniectomy (DC), and this calls for the need for monitoring of brain biochemistry for understanding these

received

June 18, 2017

accepted

July 19, 2017

published online

August 29, 2017
DOI https://doi.org/

10.1055/s-0037-1606310. ISSN 2277-954X. changes. Multimodality neurointensive monitoring is standard of care for decision making in all severe TBI cases at present in most neurotrauma units. Neuromonitoring tools, such as cerebral microdialysis, may contribute to understanding the pathomechanisms of acute brain injury, provide an early warning of secondary cerebral deterioration (therapeutic window of opportunity), and help in selecting the patients most suitable to benefit from this very invasive surgical rescue therapy (-Fig. 1). Furthermore, cerebral microdialysis can be used as a measure of the effect of DC on neurochemistry derangement and brain energy crisis jeopardizing the injured brain.

Cerebral microdialysis is a means to monitor cerebral biochemistry and has been in use globally in human studies for various conditions since the past two decades. The various compounds measured by cerebral microdialysis include the glucose, pyruvate, lactate, glycerol, glutamate, and urea.

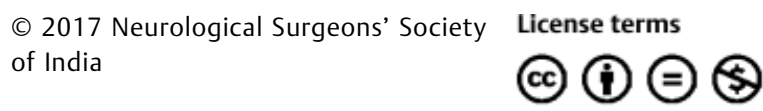




\section{What is the main clinical purpose?}

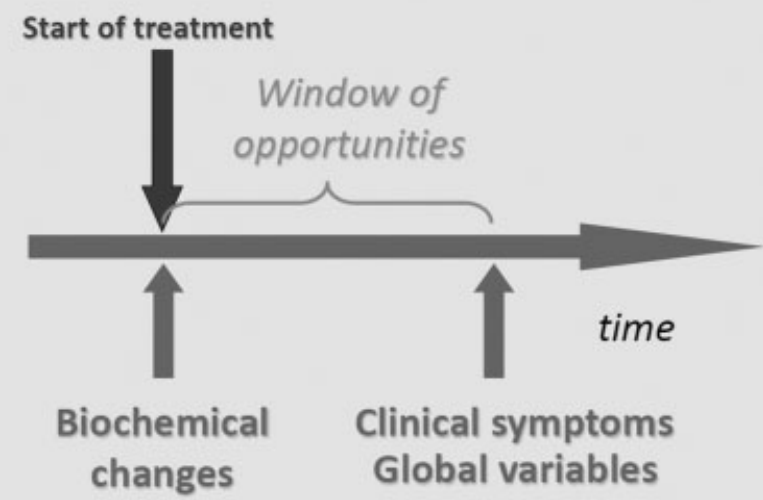

Fig. 1 Cerebral microdialysis and neuromonitoring tools offer therapeutic window of few hours before new-onset clinical symptoms.

Studies on TBI patients using microdialysis have shown that injured parts of the brain contain high lactate levels corresponding to significant acidosis.

Cerebral microdialysis can monitor brain tissue biochemical changes bedside, continuously ( 1 hourly) by means of a thin catheter inserted in the brain parenchyma, and dialysate thus obtained can be analyzed allowing real-time diagnosis of ongoing ischemia at bedside. Insertion of microdialysis catheters has been shown to be safe in many studies available and is being used for neuromonitoring already globally in advanced neurointensive units where this facility is available.

Rise in lactate (acidosis) and in lactate/pyruvate ratio (LPR) has been noted in ischemic brains especially in penumbra zone in such cases, and these biochemical changes can get altered further with worsening of ischemia/hypoxia, thereby suggesting if interventions to reduce ischemia (increase of cerebral perfusion/cerebral blood flow or decreasing ICP by medical or surgical means) are implemented well in time, outcome in such cases could be improved by improving local cerebral blood flow and decreasing ischemia. There is likely to be a therapeutic window of biochemical alterations preceding rise in $\mathrm{ICP} /$ fall in cerebral perfusion pressure (CPP) in these cases, and there exists probably a time period during which early timely interventions can be beneficial.

\section{Principle of Microdialysis}

Microdialysis is a minimally invasive technique in which a thin probe, with a semipermeable dialysis membrane at its tip, is inserted into the brain for continuous sampling of the brain interstitial fluid.

The microdialysis catheter takes up substances delivered by the blood, for example glucose and drugs, but also substances released from the cells, for example markers of cellular metabolism. The interstitial fluid is the "cross road" of all substances passing between cells and blood capillaries. The dialysis membrane at the distal end of a microdialysis catheter functions like a blood capillary. Molecules at high concentration in the extracellular fluid equilibrate across the semipermeable membrane. The enriched perfusate (i.e., the dialysate) is collected via an outlet tube and can be analyzed.

Chemical substances from the interstitial fluid diffuse across the membrane into the perfusion fluid inside the catheter. Monitoring this compartment in the brain allows the clinician to have critical information about the biochemistry of neurons and glia and how seriously brain cells are affected by, for example ischemia, hyperemia, trauma, vasospasm, as well as various physiologic and pharmacologic interventions (-Fig. 2a-d).

Depending on the availability of an appropriate analytical assay, virtually any soluble molecule in the interstitial fluid can be measured by microdialysis. Most commonly, microdialysis catheter probes (molecular weight cutoff of $20 \mathrm{kDa}, 10-\mathrm{mm}$ membrane length, and an external diameter of $0.6 \mathrm{~mm}$ ) are placed in the perilesional tissue. The catheter can also be placed via burr hole in patients being managed conservatively/ on contralateral side for normal control sample assays. The probes are perfused through a precision microdialysis pump with CNS perfusion fluid at 0.3 to $2 \mu \mathrm{L} / \mathrm{min}$ and samples collected for bedside analysis.

The "recovery" of a particular substance is defined as the concentration in the dialysate expressed as a percent of the concentration in the interstitial fluid. Factor affecting recovery of an analytes include perfusion flow rate, molecular weight cutoff of the dialysis membrane, surface area of the dialysis membrane (length and diameter of the probe), diffusion rate in dialysis membrane, blood flow rate, metabolism rate, and extent of tissue vascularization. In particular, measured values for interstitial products vary with perfusion rates; the lower the rate $(0.3 \mu \mathrm{L} / \mathrm{min})$, the higher the relative recovery of the molecule. ${ }^{1}$ Concentrations of glucose, lactate, pyruvate, glutamate, and glycerol in the microdialysate are analyzed using the microdialysis analyzer.

By the use of advanced neuromonitor tools, such as cerebral microdialysis, in the injured brain, the clinician is provided with a very early warning of secondary cerebral deterioration that offers the opportunity to deliver targeted therapy before irreversible neuronal damage occurs. Biochemical data are more informative, if integrated with other neurophysiologic parameters commonly monitored after an acute brain injury, such as ICP, CPP, mean arterial pressure (MAP), and plasma glucose.

\section{Microdialysis as a Measure of Brain Energy Metabolism Alterations, Brain Tissue Ischemia, Excitotoxicity, and Neuroinflammation after TBI}

TBI is responsible for a complex dysfunction in brain energy metabolism. Oxygen is needed for oxidative phosphorylation in the mitochondria.

Under normal aerobic conditions, glucose is converted to pyruvate by glycolysis and pyruvate is then metabolized in the citric acid cycle and the mitochondrial respiratory chain. In TBI, shear forces and the release of free radicals and intracellular calcium lead to inactivation of mitochondrial respiration and 
a
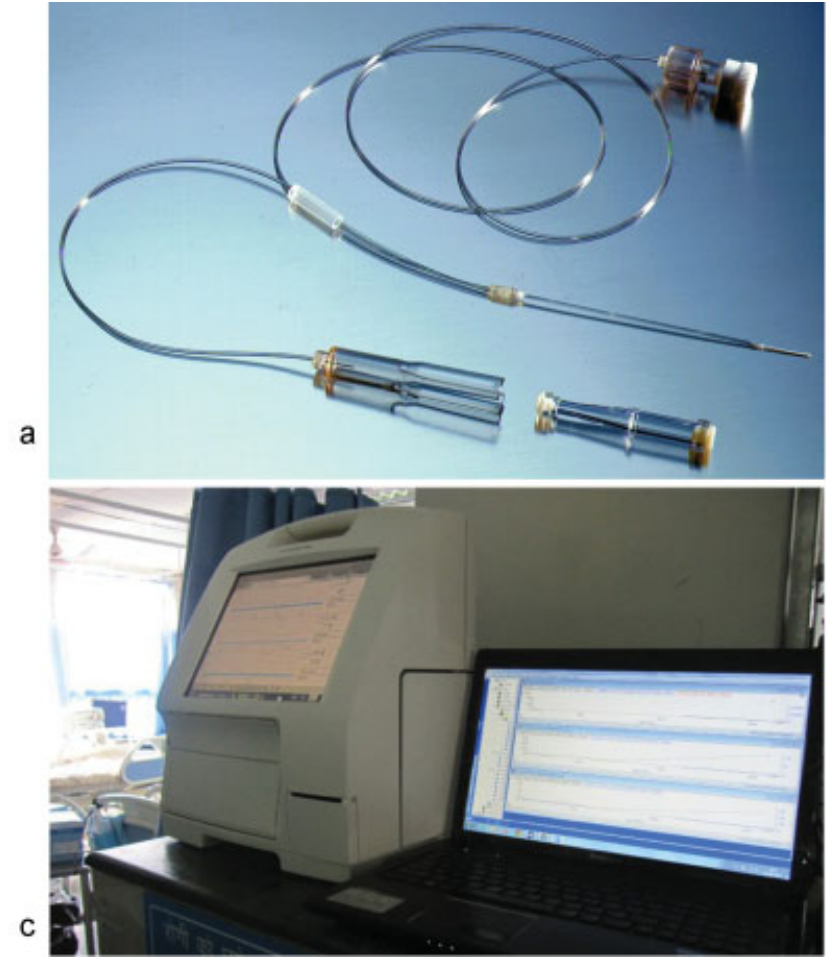

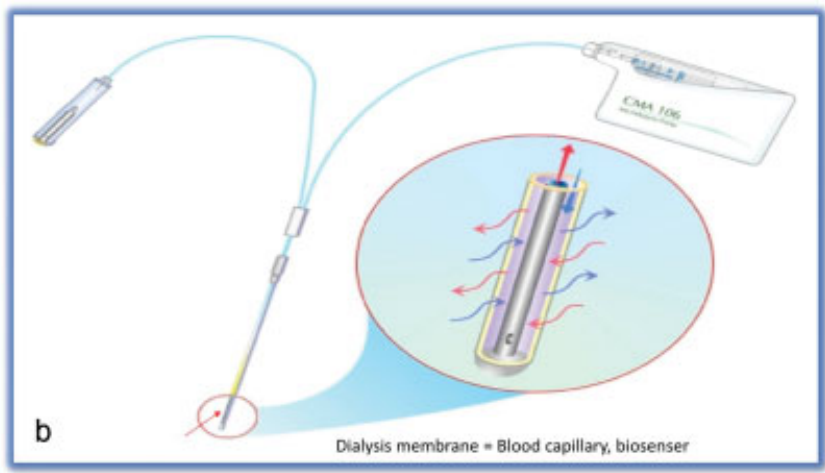

(n)

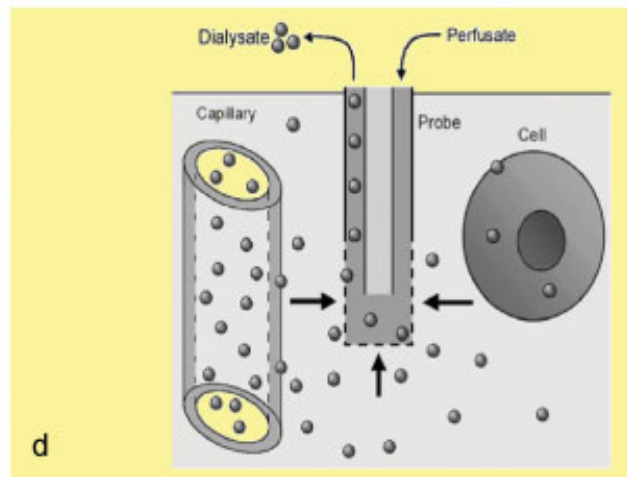

Fig. 2 (a) Cerebral microdialysis catheter and microvial used for cerebral microdialysis. (b) Microdialysis catheter as universal biosensor/blood capillary. (c) Cerebral microdialysis analyzer used to monitor the values of dialysate bedside. (d) Schematic picture showing principle of microdialysis with microdialysis in center collecting products of cellular metabolism from cell and interstitial fluid components from capillary by diffusion.

the stages of the Krebs cycle, leading to mitochondrial dysfunction and brain energy crisis. In fact, with insufficient oxygen delivery, neurons must rely on anaerobic metabolism of glucose for energy. During ischemia, there is a shift from aerobic to anaerobic metabolism, and pyruvate, unable to enter the citric acid cycle, is reduced to lactate, accepting electrons from NADH and so providing the NAD necessary for glycolysis to continue. The extracellular reflection of these intracellular events is: reduction in glucose and pyruvate and increase in lactate and LPR. The use of microdialysis allows the manifestation of this "energy crisis" ( $\mathbf{- F i g . ~ 3 ) ~ t o ~ b e ~ d e t e c t e d ~}$ and monitored over time and the effect of therapeutic intervention to be evaluated.

Glutamate is another small molecule measured by microdialysis that may be used as a marker of excitotoxicity, which is a proposed mechanism of secondary brain injury, mediated by massive calcium influx into brain cells via glutamate-mediated opening of neuronal calcium channels. ${ }^{2,3}$ Glutamate has been seen to be elevated 100 - to 200 -fold in severe primary human TBI, and in very severe secondary insults, it is correlated with herniation, hypoxia/ischemia, and poor outcome. $^{4-6}$

Glycerol is a less widely reported but well-validated marker of free radical generation, bimolecular leaflet cell membrane damage, phospholipid breakdown, and consequent free-fatty acid release. This results from hypoxic/ischemic events, as well as calcium release into neurons and astrocytes, via the inactivation of protective enzymes such as peroxidase and superoxide dismutase, among others. ${ }^{4,7,8}$
Cerebral microdialysis is a well-established research tool that has enormously contributed to the authors' understanding of the derangements occurring in the acutely injured brain, as demonstrated by a rich literature after acute brain injury in humans.

In a population of comatose-operated patients with secondary brain ischemia following aneurysmal subarachnoid hemorrhage (SAH) and severe TBI, Dizdarevic et al ${ }^{9}$ conducted a

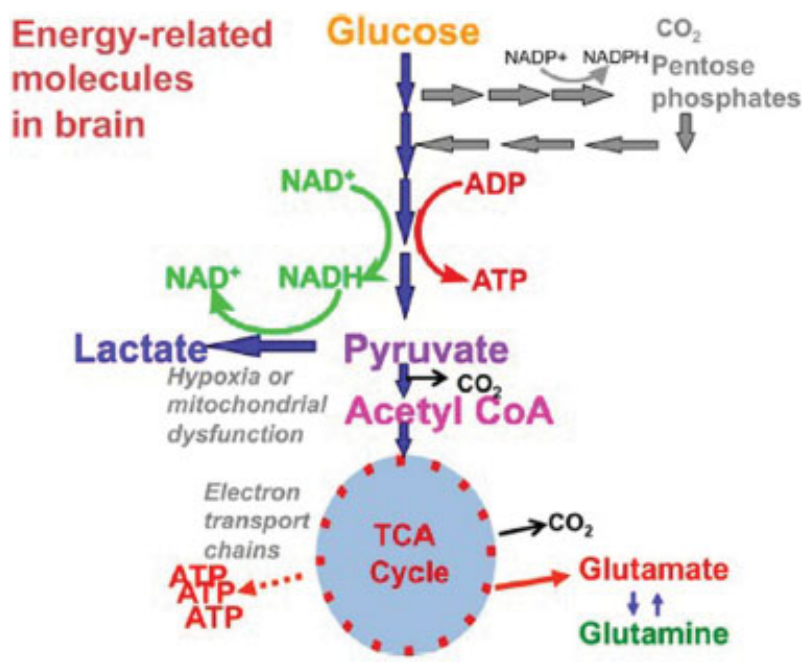

Fig. 3 Schematic representation of citric acid cycle showing energy metabolism in brain tissue. ADP, adenosine diphosphate; ATP, adenosine triphosphate; TCA, tricarboxylic acid. 
prospective, randomized controlled study comparing relative effectiveness of the two popular management strategies for head injury such as ICP-targeted therapy with cerebral microdialysis monitoring according to the modified Lund's concept or CPP-targeted therapy. The authors found that patients treated with ICP-targeted therapy with microdialysis monitoring had significantly lower mortality rate as compared with those treated with CPP-targeted therapy. Patients monitored with microdialysis who had poor outcome had lower mean values of glucose and higher mean values of glycerol and LPR as compared with those who had good outcome. There was no difference in the mortality outcome between aneurysmal SAH and severe TBI in the two groups. The authors concluded that the modified Lund's concept, directed at bedside real-time monitoring of brain biochemistry by microdialysis, showed better results compared with CPPtargeted therapy in this patient population.

Omerhodzić et $\mathrm{al}^{10}$ performed an observational prospective clinical study on the treatment and outcome of 51 patients with SAH and traumatic intracranial hemorrhage. Positive correlation was found between brain interstitial glucose level and outcome at 1-year follow-up (when glucose level was lower, the patient Glasgow outcome scale [GOS] score was worse), suggesting that early initiation of appropriate therapeutic strategies to overcome cerebral ischemia and secondary brain damage eventually leads to better patient outcome.

Karathanou et $\mathrm{al}^{11}$ investigated the usefulness of cerebral microdialysis in predicting the outcomes of 38 TBI patients and concluded that biochemical parameters analyzed using microdialysis could serve as predictor indexes of clinical outcome several months after the injury.

Rostami and Bellander ${ }^{12}$ investigated the correlation of glucose levels in peripheral blood, subcutaneous (SC) fat, and extracellular intracerebral tissue in patients with severe TBI during neurointensive care. They found a significant positive correlation between levels of glucose in peripheral blood, SC fat, and noninjured brain during the initial 12 hours but not in injured brain. They concluded that there is a good correlation between blood and adipose tissue glucose levels during initial and later time points in the neurointensive care unit whereas the correlation between blood and brain glucose levels seems to be more individualized among patients. This emphasizes the importance of using intracerebral microdialysis to ensure adequate intracerebral levels of glucose in patients suffering from severe TBI and to detect hypoglycemia in the brain despite normal levels of blood glucose.

In a study ${ }^{13}$ to determine the relationship between the fundamental biochemical markers and neurologic outcome in a large cohort of patients with TBI, it was suggested that extracellular metabolic markers as measured by microdialysis are independently associated with outcome following TBI.

Furthermore, in a large population of TBI patients, Bullock and coworkers demonstrated that the results of microdialysisbased neurochemical monitoring correlate with quantitation of ischemic events, as measured by a simple five-item scoring system, taking into account the occurrence of specific potentially brain-damaging events (hypoxemia, hypotension, low cerebral blood flow, herniation, and low CPP). ${ }^{14}$

Recently, in a population of TBI patients undergoing spreading depolarization (SD) monitoring, with electrocorticography, the association of SD and neurochemical markers as measured by microdialysis were studied. ${ }^{15}$ The authors showed that extracellular glutamate $(50 \mu \mathrm{mol} / \mathrm{L})$ and lactate $(3.7 \mathrm{mmol} / \mathrm{L})$ were significantly elevated on day 0 in patients with SD. Furthermore, glutamate and LPR progressively increased with the number of SDs, exceeding the pathologic threshold for excitotoxicity (glut $>50$ ) and metabolic crisis (LPR > 40). The same group also demonstrated a strong association between SD and the occurrence of secondary insults after TBI (low CPP, low MAP, high temperature). ${ }^{16}$ These results suggest SD as an important pathomechanism of secondary brain damage after TBI and confirm the role of microdialysis in the study of pathomechanism of brain injury.

The role of neuroinflammation as a key pathomechanism of damage after acute brain injury has also been investigated with cerebral microdialysis. Posttraumatic neuroinflammation is characterized by glial activation, leukocyte recruitment, and upregulation and secretion of mediators such as cytokines and chemokines. In particular, Helmy et al demonstrated the different pattern of response in brain and peripheral blood of cytokines production and the difference in cytokines profile after recombinant human IL-1ra administration in a phase II randomized control trial. ${ }^{17,18}$

The use of microdialysis monitoring to evaluate putative neuroprotective drugs after severe TBI has been also documented in clinical trials, demonstrating the utility of this monitoring tool, not only to measure the effect of a treatment on brain biochemistry ${ }^{19}$ but also to detect the brain interstitial level of intravenous administered neuroprotective drugs. ${ }^{20}$ This can help for safer dose optimization to achieve target concentrations of putative neuroprotectant drugs for neuroprotection in future TBI studies.

\section{Cerebral Microdialysis Usefulness in Neuromonitoring after Decompressive Craniectomy}

Neuromonitoring can be a measure of the effect of treatment (i.e., DC) on cerebral ischemia and hypoxia, as it relates to neurologic outcomes. The results of neuromonitoring can offer clinicians a perspective on the appropriate time window for the investigated rescue treatment ${ }^{21-23}$ ( - Figs. 4, 5). Placement of catheter is desired in penumbra zone peripheral to contused brain tissue or on the same side in noneloquent area after evacuation of subdural hematoma (-Fig. 6).

Guidance of critical care management after DC, especially in those cases characterized by occurrence of a hyperemic response after the procedure, may be an important next step. In fact, postoperative derangement in cerebrovascular pressure reactivity may be present after DC and could cause malignant cerebral hyperemia, as may be seen in the operating room in the case of very high CPP. ${ }^{24}$ Failure of restoration of brain energy metabolism resulting from a persistent energy metabolic crisis may occur, 


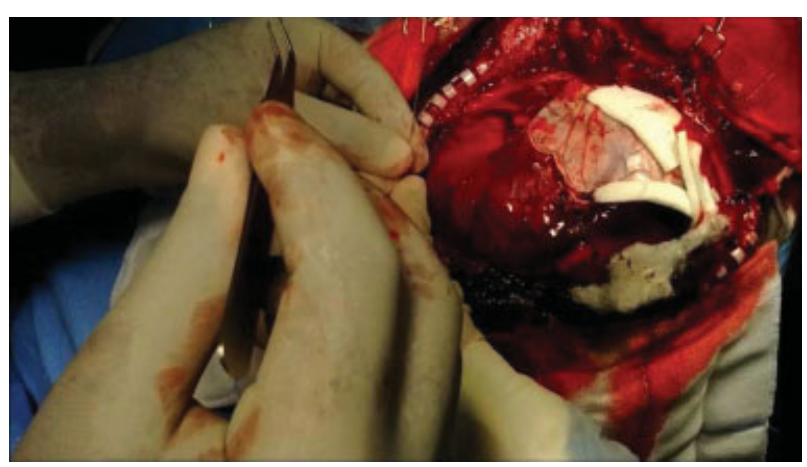

Fig. 4 Microdialysis catheter being inserted after decompressive craniectomy.

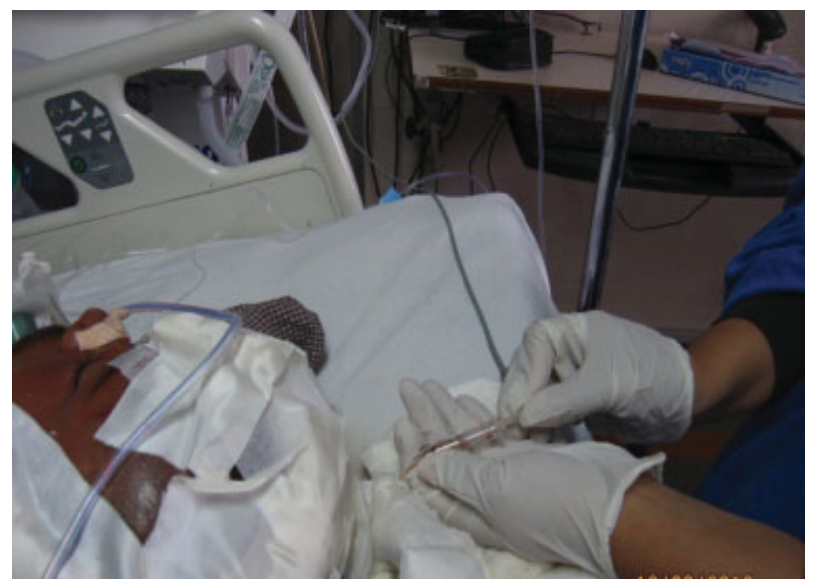

Fig. 5 Collection of dialysate bedside for neurobiochemistry of microdialysis sample.

demonstrated by mitochondrial dysfunction or signs of cerebral ischemia, and it clearly needs further evaluation. ${ }^{24}$

Overall, the evaluation of the effects of DC on neurochemistry and brain oxygen delivery may contribute to a better understanding of the role of this procedure in the treatment of intracranial hypertension.

Gupta et al ${ }^{25,26}$ studied a cohort of 41 adult patients with severe TBI undergoing DC. Twenty-six (63.4\%) patients had a favorable outcome (GOS score 4 or 5 ) and 15 patients (36.6\%) had an unfavorable outcome (GOS scores 1-3) at 6 months' follow-up.

All patients were managed in the neurointensive care unit with multimodality monitoring over a course of 5 days following DC. Total 3,813 hours of monitoring values of various microdialysis variables were obtained. There were no episodes of catheter insertion-induced hematoma or meningitis.

In these studies, the authors demonstrated significant cerebral biochemical alterations, as detected by cerebral microdialysis.

\section{Glucose}

There was no significant difference in mean random blood sugar (RBS) ( 7.26 vs. $7.61 \mathrm{mmol} / \mathrm{L} ; p=0.166)$ and mean cerebral glucose ( 1.84 vs. $1.95 \mathrm{mmol} / \mathrm{L} ; p=0.221$ ) values among the good or bad outcome groups, respectively. A poor correlation between plasma and cerebral glucose for the entire cohort was noted $(p=0.642)$. This suggests that brain interstitial microdialysate glucose values are not always reflective of changes in systemic glucose. A significant difference was noted in the incidence of hyperglycemia (RBS $>10 \mathrm{mmol} / \mathrm{L}$ ) and hypoglycemia (RBS $<5 \mathrm{mmol} / \mathrm{L}$ ) between the two outcome groups $(p<0.0001)$. The microdialysis glucose values during the episodes of hyperglycemia or hypoglycemia did not show a significant difference between the two groups. However, the authors noticed that microdialysis glucose values showed a rising trend over the course of 5 days of monitoring in the good outcome group. Similarly, the good outcome group showed rising microdialysis glucose to blood glucose ratios. This indicates a recovering metabolic state in the brain tissue that may indicate a good outcome $e^{25,26}$ (-Fig. 7).
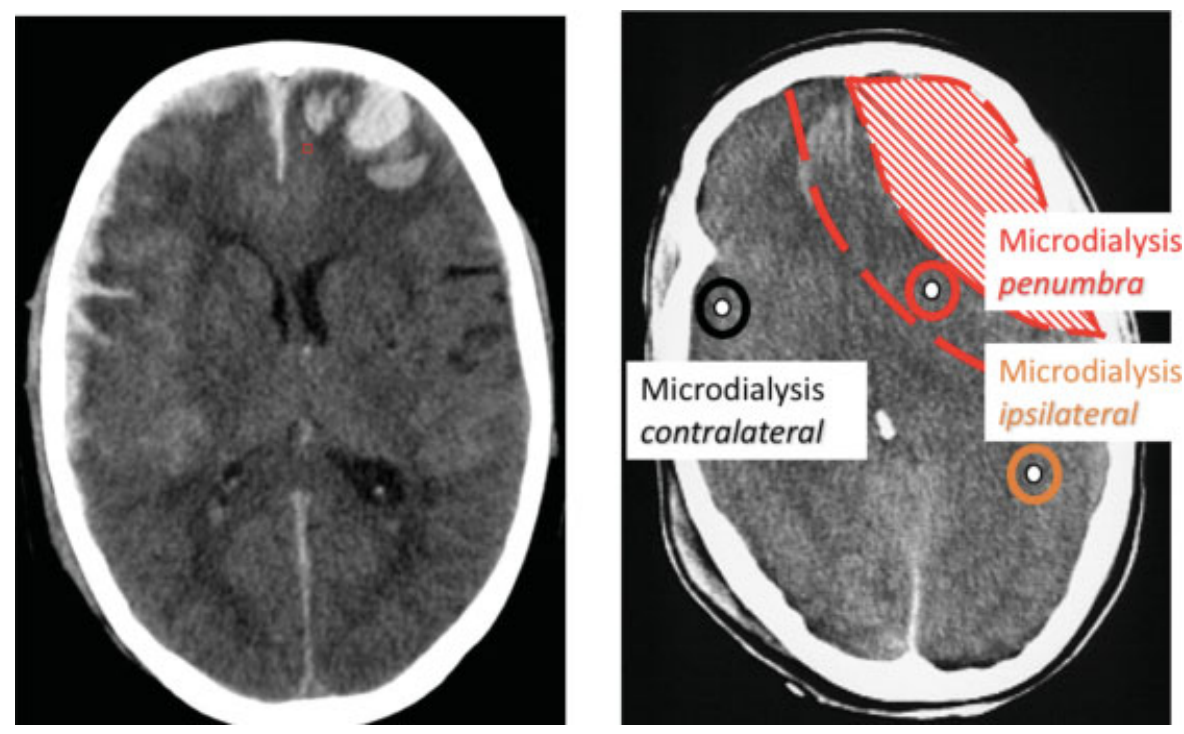

Fig. 6 Noncontrast CT showing focal hemorrhagic left frontal contusion (panel a). After removal of contusion, placement of microdialysis catheters in penumbra zone and normal contralateral (panel b). 


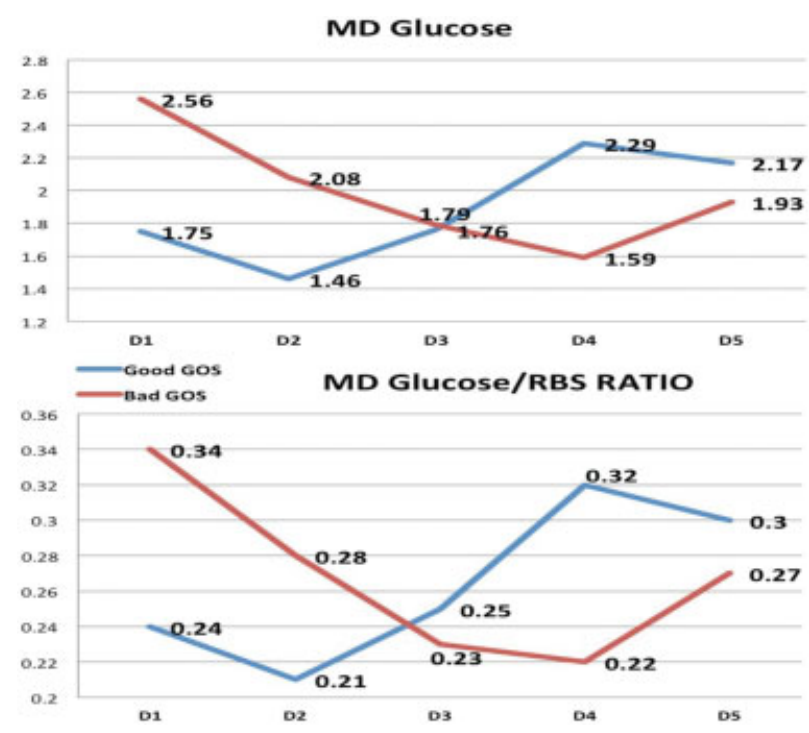

Fig. 7 Over 5 days of intracerebral microdialysis (MD) monitoring, the good Glasgow outcome scale (GOS) showed a rising cerebral MD glucose and cerebral/blood glucose ratios. MD glucose is expressed in $\mathrm{mmol} / \mathrm{L}$ and random blood glucose (RBG) in $\mathrm{mmol} / \mathrm{L}$. RBS, random blood sugar.

Difference between the two groups, comparing episodes of cerebral hypoglycemia during systemic hypoglycemic episodes (RBS $<5 \mathrm{mmol} / \mathrm{L})$, was significant $(p=0.0026)$. This probably reflects increased susceptibility of brain tissue to hypoglycemia, related to impaired autoregulation, in the poorer outcome group. The previous assumption that cerebral extracellular glucose concentration would change in parallel with blood glucose values may be an oversimplification. ${ }^{27}$ An increased metabolism of intracerebral glucose may lead to lower microdialysis glucose values following head injury. ${ }^{28}$ Similarly, poor correlation has been also reported between blood and extracellular brain glucose in patient with SAH. ${ }^{29}$ Abate and colleagues attributed poor control of microdialysis glucose based on blood glucose values secondary to the metabolic heterogeneity that may be expected in TBI patients. $^{30}$

Nagel et al noted weak correlation between cerebral and serum glucose; however, the trend of values for cerebral hypoglycemia, LPR, and CPP correlated well with outcomes in their series. ${ }^{31}$

\section{Lactate/Pyruvate Ratio}

After DC, Gupta et al showed that mean LPR was significantly higher in the poor outcome group than the good outcome group (mean 80.2 [SD 320.3] vs. 45.8 [SD 169.8]; $p<0.01$ ). CPP had an inverse correlation to the LPR for the whole cohort as would be expected with increased perfusion $(p=0.03)$. An inverse correlation was also seen in the unfavorable outcome group $(p<0.01) \cdot{ }^{25,26}$

Nikaina et al previously showed that CPP and LPR have significant effects on the long-term outcome prognosis after a spontaneous cerebral hematoma. ${ }^{32}$ They proposed a cutoff CPP of $\geq 75 \mathrm{~mm} \mathrm{Hg}$ and LPR $\leq 36$. Parafarou et al suggested a
CPP cutoff $>75 \mathrm{~mm} \mathrm{Hg}$, LPR $<37$, and glycerol $<72 \mu$ moles/L as markers of good outcome. ${ }^{23}$ This study shows somewhat similar results (LPR $<45$ and CPP $\geq 70 \mathrm{~mm} \mathrm{Hg}$ ).

In the event of TBI, ischemia and decreased cerebral blood flow can occur even with CPP $\approx 65$ to $75 \mathrm{~mm} \mathrm{Hg}{ }^{23,33}$ Therefore, CPP alone is not a reliable indicator to assess adequacy of the cerebral blood flow in such patients. There is, however, no consensus for the ideal CPP values in severe TBI. Some authors have warned about a higher risk of acute respiratory distress syndrome with a CPP $>70 \mathrm{~mm} \mathrm{Hg}$, with no additional benefit to outcome. A lower CPP goal of $60 \mathrm{~mm} \mathrm{Hg}$ has been endorsed by the American Association of Neurological Surgeons, and recently the Brain Trauma Foundation suggested a general threshold in the area of 50 to $70 \mathrm{~mm} \mathrm{Hg.}{ }^{34}$ An elevated LPR can have various causes such as ischemia, hyperglycolysis, and mitochondrial dysfunction. ${ }^{35-37}$ The key to finding the appropriate cutoff for CPP may depend on keeping a favorable metabolic profile, as indicated by low LPRs.

\section{Glutamate}

Increased levels of extracellular glutamate have been reported in human TBI, with persistent glutamate elevation seen in the postinjury period. In ischemic models, levels have been seen to rise from 2 to $>20 \mu \mathrm{mol} / \mathrm{L}$.

In the studies by Gupta et al, levels of glutamate were significantly higher in the bad outcome group than the good outcome group: $40.31 \mu \mathrm{mol} / \mathrm{L}$ (SD 67.24) versus $21.68 \mu \mathrm{mol} / \mathrm{L}$ (SD 38.31), $p<0.01 .^{24-26}$ Recovering trends of glutamate values were seen in the good outcome group over a period of 5 days, consistent with previous studies. ${ }^{38}$ This confirms that glutamate could be a useful prognostic marker in TBI.

\section{Mitochondrial Dysfunction and Ischemia}

As mentioned earlier, the LPR elevation has been seen to occur in conditions of ischemia (with a fall in pyruvate) and also in mitochondrial dysfunction (with a near normal pyruvate level). Gupta et al noted that the unfavorable outcome group showed significantly higher episodes of both mitochondrial dysfunction and ischemia than the favorable GOS group: $65.9 \%$ versus $55.9 \%(p<0.0001) ; 13.9 \%$ versus $7.24 \%$ $(p<0.001)$, respectively. ${ }^{25,26}$ These changes were more pronounced with pericontusional catheter placement. The authors compared the results for the time spent in each category, post-resuscitation GCS and age to the GOS score at last follow-up. For most patients being monitored for a period of 3 to 5 days, this meant that even a $10 \%$ increase in ischemia (8-10 hours), as shown by microdialysis, doubled their odds of progressing to a worse outcome group. Similarly, for mitochondrial dysfunction, the odds ratio was 1.475 (95\% confidence interval [CI]: 1.066-2.040) $(p=0.019)$. During episodes of ischemia, the ICP was significantly higher and the CPP significantly lower. ${ }^{36}$ In view of the odds of worsening outcome with each episode of ischemia, close control of CPP should be maintained.

Ischemia and mitochondrial dysfunction require different therapies. If a condition is characterized as 

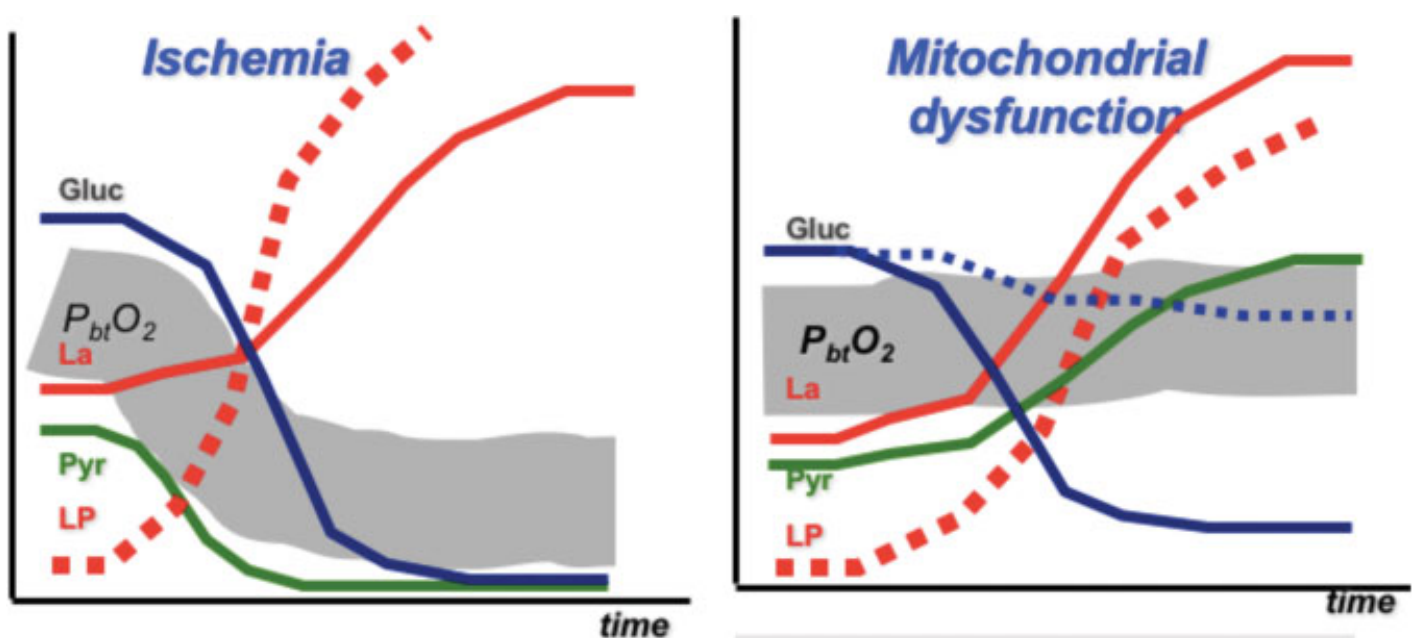

\section{Brain energy crisis without ischemia Type2 LPR elevation}

Fig. 8 Low pyruvate levels in ischemia and high pyruvate levels in mitochondrial dysfunction patients as detected by cerebral microdialysis. Lactate/ pyruvate (LP) ratio is raised in both groups. Gluc, cerebral glucose, La, cerebral lactate levels; LPR, lactate/pyruvate ratio $\mathrm{PbtO}_{2}$, brain tissue oxygenation; Pyr, cerebral pyruvate levels.

ischemic based on microdialysis values of the LPR and pyruvate levels, therapy is indicated to increase CPP or to pharmacologically improve microcirculation. On the other hand, bedside detection of nonischemic mitochondrial dysfunction should lead to consideration of novel therapies targeting mitochondrial function, such as hyperbaric oxygen $^{36}$ (-Fig. 8).
It is important to notice that in TBI shifting of energy metabolism from aerobic to anaerobic glycolysis may occur not only because of lack of oxygen delivery but also because of failure of effective utilization of the delivered oxygen due to mitochondrial dysfunction. This leads to a nonischemic LPR elevation, which has been designated type 2 elevation. This is what Vespa and colleagues have demonstrated in

Table 1 Summary of the consensus statements and recommendations regarding cerebral microdialysis

\begin{tabular}{|c|c|c|c|}
\hline & Consensus statement 2015 & Recommendation & Evidence \\
\hline $\begin{array}{l}\text { Methodology of } \\
\text { microdialysis }\end{array}$ & $\begin{array}{l}20-k D a \text { catheters for small molecules } \\
\text { and } 100-k D A \text { for large molecules }\end{array}$ & $\begin{array}{l}\text { Flow rate of } 0.3 \mu \mathrm{L} / \mathrm{min} \\
\text { with hourly sampling } \\
\text { recommended }\end{array}$ & \\
\hline \multirow[t]{5}{*}{ Reference values } & $\begin{array}{l}\text { Glucose } \\
\text { (Tier } 1 \text { intervention) }\end{array}$ & $\begin{array}{l}<0.2 \mathrm{mmol} / \mathrm{L} \\
<0.8 \mathrm{mmol} / \mathrm{L}\end{array}$ & $\begin{array}{l}\text { Low glucose associated with } \\
\text { poor outcome }\end{array}$ \\
\hline & $\begin{array}{l}\text { L/P ratio } \\
\text { (Tier } 1 \text { intervention) }\end{array}$ & $\begin{array}{l}>40 \\
>25\end{array}$ & $\begin{array}{l}\text { High L/P ratio associated with } \\
\text { poor outcome }\end{array}$ \\
\hline & Lactate & $>4 \mathrm{mmol} / \mathrm{L}$ & $\mathrm{L} / \mathrm{P}$ ratio better than lactate \\
\hline & $\begin{array}{l}\text { Glutamate } \\
\text { (Tier 2) }\end{array}$ & Raised in ischemia & May be useful in prognostication \\
\hline & $\begin{array}{l}\text { Glycerol } \\
\text { (Tier 3) }\end{array}$ & Limited specificity & No definitive evidence \\
\hline \multirow[t]{2}{*}{$\begin{array}{l}\text { Management } \\
\text { guidelines }\end{array}$} & Glucose & \multicolumn{2}{|l|}{$\begin{array}{l}\text { Raise RBS if low } \\
\text { Treat perfusion, if low CPP }\end{array}$} \\
\hline & L/P ratio & \multicolumn{2}{|l|}{$\begin{array}{l}\text { If ischemia, raise CPP } \\
\text { If low } \mathrm{PbtO}_{2} \text {, } \\
\text { improve oxygenation }\end{array}$} \\
\hline \multirow[t]{2}{*}{$\begin{array}{l}\text { Catheter } \\
\text { placement }\end{array}$} & Focal injury & $\begin{array}{l}\text { a) Ipsilateral } \\
\text { b) In normal brain }\end{array}$ & \multirow[t]{2}{*}{ Multiple catheters may be used } \\
\hline & Diffuse injury & Right frontal lobe & \\
\hline Monitoring & \multicolumn{3}{|c|}{ Trend is more important than absolute point values } \\
\hline
\end{tabular}

Abbreviations: CPP, cerebral perfusion pressure; L/P ratio, lactate/pyruvate ratio; $\mathrm{PbtO}_{2}$, brain tissue oxygenation; RBS, random blood sugar. 
their combined MD and positron emission tomography (PET) study, in which MD data revealed a $25 \%$ incidence of LPR $>40$ but only a $2.4 \%$ incidence rate of ischemia, as indicated by LPR $>40$ and glucose $<200 \mu \mathrm{mol} / \mathrm{L}$ or PEToxygen extraction fraction $>0.75 .^{37}$ In addition, LPR elevations correlated with reduced cerebral metabolic rate for oxygen, which is a measure of mitochondrial oxidative metabolism, suggesting that LPR is an indicator of mitochondrial dysfunction. This observation suggests the importance of using other markers of cell damage (glycerol and/or glutamate) in addition to LPR for a correct interpretation of this variable.

\section{Consensus Guidelines on Use of Cerebral Microdialysis}

A recently held consensus meeting by the international microdialysis forum, led by P. J. Hutchinson, combined literature review with expert opinion to produce practical guidance for the use of cerebral microdialysis (- Table $\mathbf{1}$ ). ${ }^{38}$

\section{Conclusion}

Among the several neuromonitoring tools available nowadays, cerebral microdialysis seems to accomplish most of the queries that researchers and clinicians ask to these devices in the care of patients with acute brain injury: to detect secondary insults in real time, at the time in which they can still be corrected; to clarify pathomechanisms of injury; to understand alterations in cerebral energy metabolism; to evaluate the effect of medical or surgical interventions on brain metabolism; to evaluate the effect of invasive rescue therapies such as DC in patients with intractable intracranial hypertension; to provide markers of effectiveness of therapeutic intervention; and to help in designing of clinical studies.

Therefore, monitoring neurochemistry by microdialysis can possibly provide clinicians with additional information in several related aspects of management. Robust neurochemical changes have been detected in response to clinical pathophysiologic events after an acute brain injury, so that the specific effect of DC on the different pathomechanisms of injury can be difficult to isolate from primary injury and other therapies using these neuromonitoring tools. Although cerebral microdialysis and other types of multimodality monitoring have been in use for almost two decades, their potential is huge for the future. Limits of this monitoring tool are represented especially by its high cost, its local aspect, and by the need to experienced personnel, so that its use is not widely diffused across the globe. Therefore, even if no doubt exists on its usefulness as research tool, as demonstrated by two decades of literature in human TBI, the real impact of this monitoring in dictating specific changes in management during daily clinical practice remains to be demonstrated in larger studies.

\section{Conflict of Interest}

None.

\section{Acknowledgments}

The authors wish to thank Dr. Raghav Singla (Resident Neurosurgery) and Staff Nurse Jyoti Sohal (JPNATC) for their assistance during neurobiochemistry monitoring by cerebral microdialysis in TBI patients.

\section{References}

1 Reinstrup P, Ståhl N, Mellergård P, Uski T, Ungerstedt U, Nordström $\mathrm{CH}$. Intracerebral microdialysis in clinical practice: baseline values for chemical markers during wakefulness, anesthesia, and neurosurgery. Neurosurgery 2000;47(03): 701-709, discussion 709-710

2 Bullock R, Zauner A, Myseros JS, Marmarou A, Woodward JJ, Young HF. Evidence for prolonged release of excitatory amino acids in severe human head trauma. Relationship to clinical events. Ann N Y Acad Sci 1995;765:290-297, discussion 298

3 Bullock R, Zauner A, Woodward JJ, et al. Factors affecting excitatory amino acid release following severe human head injury. J Neurosurg 1998;89(04):507-518

4 Hlatky R, Valadka AB, Goodman JC, Contant CF, Robertson CS. Patterns of energy substrates during ischemia measured in the brain by microdialysis. J Neurotrauma 2004;21(07):894-906

5 Hutchinson PJ, Gupta AK, Fryer TF, et al. Correlation between cerebral blood flow, substrate delivery, and metabolism in head injury: a combined microdialysis and triple oxygen positron emission tomography study. J Cereb Blood Flow Metab 2002; 22(06):735-745

6 Menzel M, Doppenberg EM, Zauner A, Soukup J, Reinert MM, Bullock R. Increased inspired oxygen concentration as a factor in improved brain tissue oxygenation and tissue lactate levels after severe human head injury. J Neurosurg 1999;91(01):1-10

7 Hillered L, Valtysson J, Enblad P, Persson L. Interstitial glycerol as a marker for membrane phospholipid degradation in the acutely injured human brain. J Neurol Neurosurg Psychiatry 1998; 64(04):486-491

8 Merenda A, Gugliotta M, Holloway R, et al. Validation of brain extracellular glycerol as an indicator of cellular membrane damage due to free radical activity after traumatic brain injury. J Neurotrauma 2008;25(05):527-537

9 Dizdarevic K, Hamdan A, Omerhodzic I, Kominlija-Smajic E. Modified Lund concept versus cerebral perfusion pressuretargeted therapy: a randomised controlled study in patients with secondary brain ischaemia. Clin Neurol Neurosurg 2012; 114(02):142-148

10 Omerhodzić I, Dizdarević K, Rotim K, et al. Cerebral microdialysis: perioperative monitoring and treatment of severe neurosurgical patient. Acta Clin Croat 2011;50(01):13-20

11 Karathanou A, Paterakis K, Pakopoulou M, et al. Biochemical markers analyzed using microdialysis and traumatic brain injury outcomes. J Neurosurg Sci 2011;55(03):173-177

12 Rostami E, Bellander BM. Monitoring of glucose in brain, adipose tissue, and peripheral blood in patients with traumatic brain injury: a microdialysis study. J Diabetes Sci Technol 2011;5(03): 596-604

13 Timofeev I, Carpenter KL, Nortje J, et al. Cerebral extracellular chemistry and outcome following traumatic brain injury: a microdialysis study of 223 patients. Brain 2011;134(Pt 2): 484-494

14 Mazzeo AT, Kunene NK, Choi S, Gilman C, Bullock RM. Quantitation of ischemic events after severe traumatic brain injury in humans: a simple scoring system. J Neurosurg Anesthesiol 2006;18(03):170-178

15 Hinzman JM, Wilson JA, Mazzeo AT, Bullock MR, Hartings JA. Excitotoxicity and metabolic crisis are associated with spreading depolarizations in severe traumatic brain injury patients. J Neurotrauma 2016;33(19):1775-1783 
16 Hartings JA, Strong AJ, Fabricius M, et al; Co-Operative Study of Brain Injury Depolarizations. Spreading depolarizations and late secondary insults after traumatic brain injury. J Neurotrauma 2009;26(11):1857-1866

17 Helmy A, Carpenter KL, Menon DK, Pickard JD, Hutchinson PJ. The cytokine response to human traumatic brain injury: temporal profiles and evidence for cerebral parenchymal production. J Cereb Blood Flow Metab 2011;31(02):658-670

18 Helmy A, Guilfoyle MR, Carpenter KL, Pickard JD, Menon DK, Hutchinson PJ. Recombinant human interleukin-1 receptor antagonist in severe traumatic brain injury: a phase II randomized control trial. J Cereb Blood Flow Metab 2014;34(05): 845-851

19 Mazzeo AT, Alves OL, Gilman CB, et al. Brain metabolic and hemodynamic effects of cyclosporin A after human severe traumatic brain injury: a microdialysis study. Acta Neurochir (Wien) 2008;150(10):1019-1031, discussion 1031

20 Brophy GM, Mazzeo AT, Brar S, et al. Exposure of cyclosporin A in whole blood, cerebral spinal fluid, and brain extracellular fluid dialysate in adults with traumatic brain injury. J Neurotrauma 2013;30(17):1484-1489

21 Friess SH, Lapidus JB, Brody DL. Decompressive craniectomy reduces white matter injury after controlled cortical impact in mice. J Neurotrauma 2015;32(11):791-800

22 Zweckberger K, Erös C, Zimmermann R, Kim SW, Engel D, Plesnila $\mathrm{N}$. Effect of early and delayed decompressive craniectomy on secondary brain damage after controlled cortical impact in mice. J Neurotrauma 2006;23(07):1083-1093

23 Paraforou T, Paterakis K, Fountas K, et al. Cerebral perfusion pressure, microdialysis biochemistry and clinical outcome in patients with traumatic brain injury. BMC Res Notes 2011; 4:540

24 Bor-Seng-Shu E, Figueiredo EG, Fonoff ET, Fujimoto Y, Panerai RB, Teixeira MJ. Decompressive craniectomy and head injury: brain morphometry, ICP, cerebral hemodynamics, cerebral microvascular reactivity, and neurochemistry. Neurosurg Rev 2013;36(03): 361-370

25 Gupta DK, Singla R, Kale SS, Sharma BS. Intracerebral hypoglycemia and its clinical relevance as a prognostic indicator in severe traumatic brain injury: a cerebral microdialysis study from India. Neurol India 2016;64(02):259-264

26 Singla R, Gupta D, Borkar SA, Suri A, Kale Shashank S, Sharma BS. Cerebral perfusion pressure in severe traumatic brain injury and its relation to microdialysis assessed interstitial brain glycerol and lactate pyruvate ratio. Indian J Neurotrauma 2016;13(02):59-65
27 Choi IY, Lee SP, Kim SG, Gruetter R. In vivo measurements of brain glucose transport using the reversible Michaelis-Menten model and simultaneous measurements of cerebral blood flow changes during hypoglycemia. J Cereb Blood Flow Metab 2001; 21(06):653-663

28 Vespa PM, McArthur D, O'Phelan K, et al. Persistently low extracellular glucose correlates with poor outcome 6 months after human traumatic brain injury despite a lack of increased lactate: a microdialysis study. J Cereb Blood Flow Metab 2003; 23(07):865-877

29 Schlenk F, Nagel A, Graetz D, Sarrafzadeh AS. Hyperglycemia and cerebral glucose in aneurysmal subarachnoid hemorrhage. Intensive Care Med 2008;34(07):1200-1207

30 Abate MG, Trivedi M, Fryer TD, et al. Early derangements in oxygen and glucose metabolism following head injury: the ischemic penumbra and pathophysiological heterogeneity. Neurocrit Care 2008;9(03):319-325

31 Nagel A, Graetz D, Vajkoczy P, Sarrafzadeh AS. Decompressive craniectomy in aneurysmal subarachnoid hemorrhage: relation to cerebral perfusion pressure and metabolism. Neurocrit Care 2009;11(03):384-394

32 Nikaina I, Paterakis K, Paraforos G, et al. Cerebral perfusion pressure, microdialysis biochemistry, and clinical outcome in patients with spontaneous intracerebral hematomas. J Crit Care 2012;27(01):83-88

33 Coles JP, Steiner LA, Johnston AJ, et al. Does induced hypertension reduce cerebral ischaemia within the traumatized human brain? Brain 2004;127(Pt 11):2479-2490

34 Brain Trauma F, American Association of Neurological S, Congress of Neurological S. Guidelines for the management of severe traumatic brain injury. J Neurotrauma 2007;24(Suppl 1):S1-S106

35 Oddo M, Levine JM, Frangos S, et al. Brain lactate metabolism in humans with subarachnoid hemorrhage. Stroke 2012;43(05): 1418-1421

36 Yokobori S, Mazzeo AT, Gajavelli S, Bullock MR. Mitochondrial neuroprotection in traumatic brain injury: rationale and therapeutic strategies. CNS Neurol Disord Drug Targets 2014; 13(04):606-619

37 Vespa P, Bergsneider M, Hattori N, et al. Metabolic crisis without brain ischemia is common after traumatic brain injury: a combined microdialysis and positron emission tomography study. J Cereb Blood Flow Metab 2005;25(06):763-774

38 Hutchinson PJ, Jalloh I, Helmy A, et al. Consensus statement from the 2014 International Microdialysis Forum. Intensive Care Med 2015;41(09):1517-1528 